This document is confidential and is proprietary to the American Chemical Society and its authors. Do not copy or disclose without written permission. If you have received this item in error, notify the sender and delete all copies.

\title{
Using Paramagnetism to Slow Down Nuclear Relaxation in Protein NMR
}

\begin{tabular}{|r|l|}
\hline Journal: & The Journal of Physical Chemistry Letters \\
\hline Manuscript ID & jz-2016-02417y.R1 \\
\hline Manuscript Type: & Letter \\
\hline Date Submitted by the Author: & n/a \\
\hline Complete List of Authors: & $\begin{array}{l}\text { Orton, Henry; Australian National University, Research School of Chemistry } \\
\text { Kuprov, Ilya; University of Durham, Chemistry Department } \\
\text { Loh, Choy-Theng; Australian National University, Research School of } \\
\text { Chemistry } \\
\text { Otting, Gottfried; Australian National University, Research School of } \\
\text { Chemistry }\end{array}$ \\
\hline
\end{tabular}

SCHOLARONE ${ }^{m}$

Manuscripts 


\title{
Using Paramagnetism to Slow Down Nuclear
}

\section{Relaxation in Protein NMR}

\author{
Henry W. Orton ${ }^{1}$, Ilya Kuprov ${ }^{2}$, Choy-Theng Loh ${ }^{1}$, Gottfried Otting ${ }^{1 *}$ \\ ${ }^{1}$ Research School of Chemistry, Australian National University, Canberra, ACT 2601, Australia \\ ${ }^{2}$ School of Chemistry, University of Southampton, Southampton, SO17 1BJ, United Kingdom

\section{AUTHOR INFORMATION} \\ Corresponding Author \\ *Gottfried Otting \\ e-mail: gottfried.otting@anu.edu.au
}




\section{ABSTRACT}

Paramagnetic metal ions accelerate nuclear spin relaxation; this effect is widely used for distance measurement and called paramagnetic relaxation enhancement (PRE). Theoretical predictions established that, under special circumstances, it is also possible to achieve a reduction in nuclear relaxation rates (negative PRE). This situation would occur if the mechanism of nuclear relaxation in the diamagnetic state is counterbalanced by a paramagnetic relaxation mechanism caused by the metal ion. Here we report the first experimental evidence for such a crosscorrelation effect. Using a uniformly ${ }^{15} \mathrm{~N}$-labeled mutant of calbindin $\mathrm{D}_{9 \mathrm{k}}$ loaded with either $\mathrm{Tm}^{3+}$ or $\mathrm{Tb}^{3+}$, reduced $R_{1}$ and $R_{2}$ relaxation rates of backbone ${ }^{15} \mathrm{~N}$ spins were observed compared with the diamagnetic reference (the same protein loaded with $\mathrm{Y}^{3+}$ ). The effect arises from the compensation of the chemical shift anisotropy tensor by the anisotropic dipolar shielding generated by the unpaired electron spin.

\section{TOC GRAPHICS}

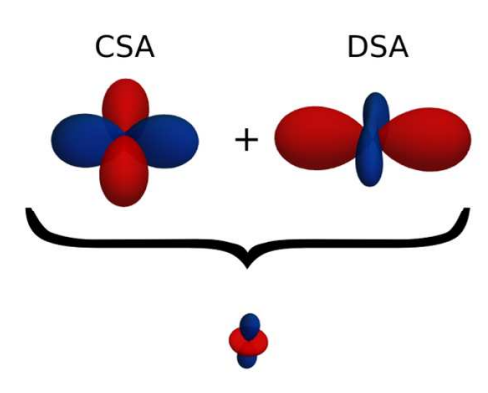

KEYWORDS ${ }^{15} \mathrm{~N}$ relaxation; calbindin $\mathrm{D}_{9 \mathrm{k}}$; CSA-DSA cross-correlation; lanthanide ions; nuclear magnetic resonance; paramagnetic relaxation enhancement. 
Paramagnetic relaxation enhancements (PRE) have long been used as powerful long-range distance restraints for structural investigations of proteins by NMR spectroscopy. ${ }^{1,2}$ In general, paramagnetic centres can be introduced using tags with unpaired electrons in either organic radicals (e.g. nitroxides) or paramagnetic metal ions. ${ }^{3,4}$ PREs generated by metal ions with isotropic magnetic susceptibility and long electron relaxation times, such as $\mathrm{Mn}^{2+}$ and $\mathrm{Gd}^{3+}$, are readily predicted by the dipole-dipole relaxation mechanism first described by Solomon. ${ }^{5}$ Many paramagnetic metal ions, however, have fast electron relaxation and anisotropic magnetic susceptibilities. ${ }^{6}$ When the electron relaxation occurs on a time scale much faster than the rotational correlation time of the molecule, the net magnetic moment of the unpaired electrons is described by the "Curie spin", which accelerates relaxation of the neighbouring nuclei. ${ }^{7}$ Curie spin relaxation, rather than Solomon relaxation, is often the dominant contribution to the PRE observed in macromolecules in solution at high magnetic field strength. ${ }^{8,9}$

The Curie spin contribution can be described by the anisotropic dipolar shielding (DSA) tensor at the site of the nuclear spin. ${ }^{10}$ Like the chemical shift anisotropy (CSA) tensor, the DSA tensor describes how the local magnetic field at the nucleus depends on the molecular orientation with respect to the external magnetic field $B_{0}$.

It has long been recognized that DSA and dipole-dipole (DD) effects between nuclear spins are modulated in the same way by the rotation of the molecule, resulting in DSA-DD crosscorrelation effects that are manifested in differential line widths for, e.g., multiplet components in homonuclear ${ }^{1} \mathrm{H}$ NMR spectra ${ }^{11}$ or the doublet components observed for a ${ }^{1} \mathrm{H}$ spin coupled to ${ }^{15} \mathrm{~N}^{12-14}$ or ${ }^{13} \mathrm{C}^{15}$. The effect depends on the distance and orientation of the internuclear vector with respect to the paramagnetic center. Under the right circumstances, it can narrow half of the multiplet components compared with a corresponding diamagnetic sample while broadening the 
other half. DSA-DD cross-correlation has no net influence on the overall longitudinal and transverse relaxation rates $R_{1}$ and $R_{2}$ respectively.

Rotational tumbling of the molecule also modulates the CSA in the same way as the DSA. Therefore, CSA-DSA cross-correlation effects are expected. ${ }^{8}$ Depending on the relative orientation of the two tensors, the cross-correlation effect is predicted to either enhance or reduce the overall nuclear relaxation rate. If the relaxation rate is reduced, slower relaxation is expected in the presence of the paramagnetic centre than in its absence (negative PRE). The term "paramagnetically effected narrowing" (PEN) has been coined to describe this phenomenon. ${ }^{8}$ To the best of our knowledge, however, no experimental observations of this effect have been reported to date, suggesting that it may be insignificant. In the case of amide protons, this may be attributed to the small contribution of CSA relaxation to the overall relaxation rate compared with the dominant dipole-dipole relaxation, to the difficulty of measuring ${ }^{1} \mathrm{H}$ relaxation rates without interference from cross-relaxation effects or coupling evolution, and to the fact that CSA tensor magnitudes and orientations of amide protons are variable and difficult to predict. ${ }^{16}$

Here we show that CSA-DSA cross-correlation effects are clearly manifested in the overall ${ }^{15} \mathrm{~N}$ relaxation rates $R_{1}$ and $R_{2}$ of paramagnetic metalloproteins. Negative PREs are readily observed because backbone amide ${ }^{15} \mathrm{~N}$ spins have large CSA tensors that strongly contribute to the overall relaxation. These CSA tensors can also be predicted from the structure with good accuracy. The negative PRE effect can be measured even though Curie spin relaxation depends quadratically on the gyromagnetic ratio $\gamma$, which is much smaller for ${ }^{15} \mathrm{~N}$ than ${ }^{1} \mathrm{H}$.

NMR data were recorded for the P43M/N56D double mutant of calbindin $\mathrm{D}_{9 \mathrm{k}}$. The protein has two binding sites for $\mathrm{Ca}^{2+}$, one of which is readily replaced by a lanthanide ion. ${ }^{17}$ The Pro43Met mutation was introduced to prevent proline cis-trans isomerisation ${ }^{12}$ and the Asn56Asp mutation 
was designed to prevent any backbone rearrangement. ${ }^{18}$ The protein was loaded with $\mathrm{Ca}^{2+}$ and either $\mathrm{Tm}^{3+}, \mathrm{Tb}^{3+}$, or $\mathrm{Y}^{3+}$; the sample with $\mathrm{Y}^{3+}$ acted as the diamagnetic reference. In the following, these samples are referred to as $\mathrm{CbCaTm}, \mathrm{CbCaTb}$, and $\mathrm{CbCaY}$, respectively. $\left[{ }^{15} \mathrm{~N},{ }^{1} \mathrm{H}\right]-\mathrm{HSQC}$ spectra displayed pseudocontact shifts (PCS; Figure 1). The PCS values for the backbone amide protons were used to fit magnetic susceptibility anisotropy $(\Delta \chi)$ tensors to the crystal structure of calbindin $\mathrm{D}_{9 \mathrm{k}}$. The $\Delta \chi$ tensor fits were of high quality, as indicated by small quality factors (Table 1), confirming the binding of the lanthanide ions to site II of the protein.

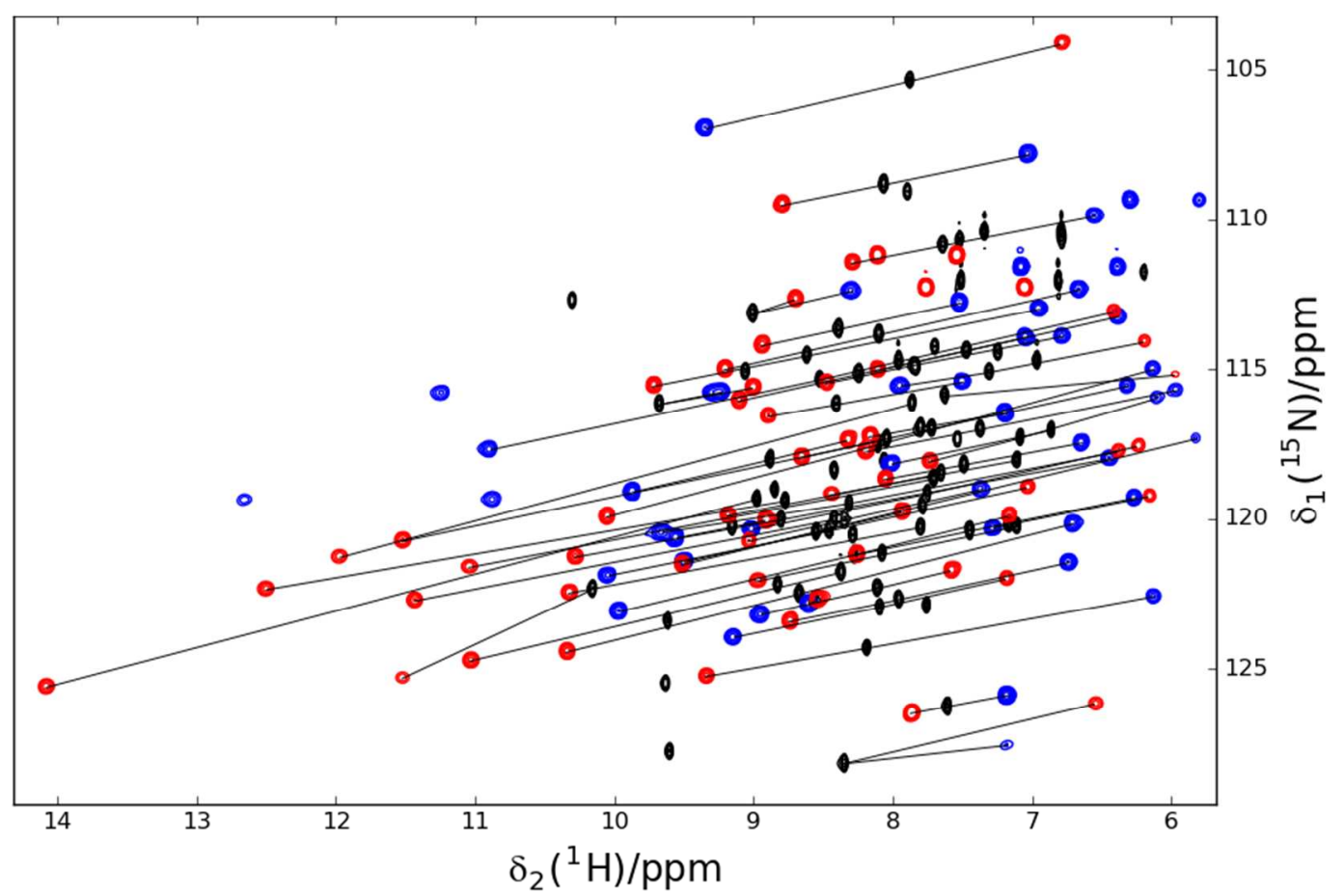

Figure 1. $\left[{ }^{15} \mathrm{~N},{ }^{1} \mathrm{H}\right]-\mathrm{HSQC}$ spectra of calbindin $\mathrm{D}_{9 \mathrm{k}} \mathrm{P} 43 \mathrm{M} / \mathrm{N} 56 \mathrm{D}$ loaded with $\mathrm{Y}^{3+}$ (black), $\mathrm{Tm}^{3+}$ (red), or $\mathrm{Tb}^{3+}$ (blue). The spectra were measured at $\mathrm{pH} 6.5$ and $25{ }^{\circ} \mathrm{C}$ at a ${ }^{1} \mathrm{H}$ NMR frequency of $600 \mathrm{MHz}$. Black lines are connecting cross-peaks belonging to the same amino acid residue. PCSs were measured as the chemical shift in the paramagnetic state minus the chemical shift in the diamagnetic state. 
Table 1. $\Delta \chi$ tensors for calbindin $\mathrm{D}_{9 \mathrm{k}} \mathrm{P} 43 \mathrm{M} / \mathrm{N} 56 \mathrm{D}^{a}$

\begin{tabular}{|l|c|c|c|c|c|c|}
\hline $\mathbf{L n}^{3+}$ & $\Delta \chi_{\mathbf{a x}} / \mathbf{1 0}^{-\mathbf{3 2}} \mathbf{m}^{\mathbf{3}}$ & $\Delta \chi_{\mathbf{r h}} / \mathbf{1 0}^{-\mathbf{3 2}} \mathbf{m}^{\mathbf{3}}$ & $\boldsymbol{\alpha} /{ }^{\circ}$ & $\boldsymbol{\beta} /{ }^{\circ}$ & $\boldsymbol{\gamma} /{ }^{\circ}$ & Q-factor \\
\hline $\mathrm{Tm}^{3+}$ & -20.4 & -9.2 & 125 & 140 & 65 & 0.05 \\
\hline $\mathrm{Tb}^{3+}$ & 29.7 & 12.5 & 155 & 152 & 75 & 0.08 \\
\hline
\end{tabular}

${ }^{a}$ The position of the lanthanide ion was constrained to site II of the crystal structure (PDB ID: $4 \mathrm{ICB})^{19}$ at $[25.79,9.52,6.56] \AA . \alpha, \beta$, and $\gamma$ are Euler angles as determined using the program Numbat. $^{20}$

Longitudinal relaxation rates $R_{1}$ were measured for the backbone ${ }^{15} \mathrm{~N}$ nuclei using a standard 2D HSQC type pulse sequence (see Figure S2 in the Supporting Information, SI). Transverse relaxation rates $R_{2}$ were measured with a similar HSQC type pulse sequence using a single spinecho delay (Figure S3). The measurements were performed for the paramagnetic samples $(\mathrm{CbCaTb}$ and $\mathrm{CbCaTm})$ and the diamagnetic reference $(\mathrm{CbCaY})$. Experimental PREs were calculated as differences between relaxation rates measured in the paramagnetic state and the corresponding rates in the diamagnetic state.

Theoretical predictions (see the SI) for the PREs were based on the crystal structure (PDB ID: 4ICB), ${ }^{19}$ average ${ }^{15} \mathrm{~N}$ CSA parameters determined for ubiquitin, ${ }^{21}$ and the $\Delta \chi$ values listed in Table 1. The PREs were predicted in two different ways: either considering only Curie spin (DSA) relaxation generated by a lanthanide ion with an anisotropic magnetic susceptibility, ${ }^{22}$ or by including the CSA-DSA cross-correlation effect. When the cross-correlation is ignored, only positive PREs are predicted. When the cross-correlation is included, negative PREs are predicted for the ${ }^{15} \mathrm{~N}$ sites where the anisotropy of the CSA tensor is counteracted by the anisotropy of the DSA tensor. 

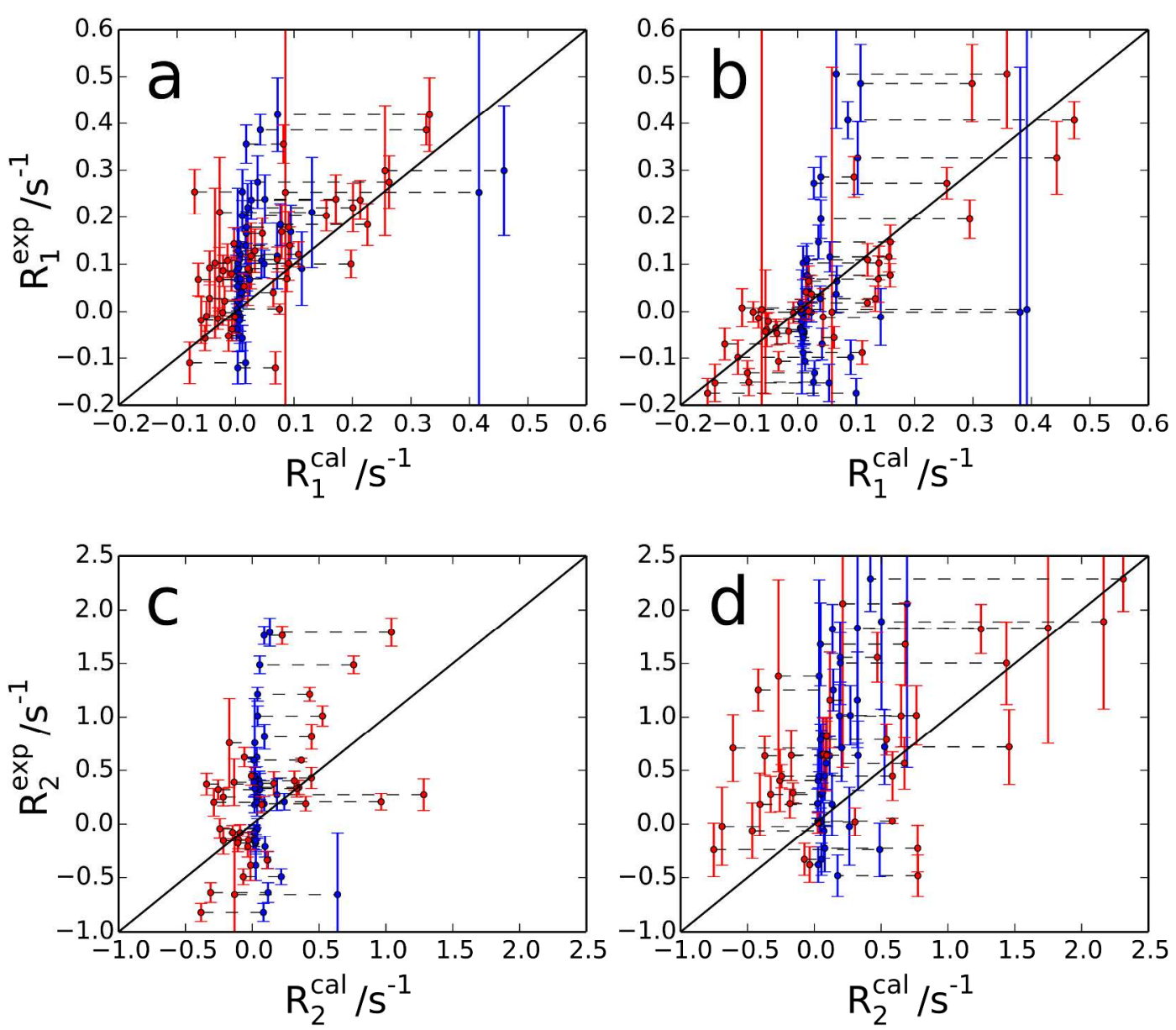

Figure 2. Experimental $R_{1}\left({ }^{15} \mathrm{~N}\right)$ and $R_{2}\left({ }^{15} \mathrm{~N}\right)$ PREs in calbindin $\mathrm{D}_{9 \mathrm{k}}$ versus predictions made by considering only Curie spin relaxation (blue points), ${ }^{22}$ and by including CSA-DSA crosscorrelation (red points, this work). Corresponding data points for the same amino acid residue are connected by dashed lines. (a) $R_{1}\left({ }^{15} \mathrm{~N}\right)$ PREs of CbCaTm. (b) $R_{1}\left({ }^{15} \mathrm{~N}\right)$ PREs of CbCaTb. (c) $R_{2}\left({ }^{15} \mathrm{~N}\right)$ PREs of CbCaTm. (d) $R_{2}\left({ }^{15} \mathrm{~N}\right)$ PREs of CbCaTb. See Figure $\mathrm{S} 1$ for plots of the full data range in (a) and (c).

The correlation between the experimental and the calculated relaxation rates clearly shows the importance of taking into account the CSA-DSA cross-correlation effect (Figure 2). Negative 
$R_{1}\left({ }^{15} \mathrm{~N}\right)$ PREs were experimentally observed for a number of residues both in CbCaTm and $\mathrm{CbCaTb}$ (Figure 2a and c). Although the experimental evidence for negative $R_{2}\left({ }^{15} \mathrm{~N}\right)$ PREs was less obvious, the agreement improves significantly when the cross-correlation effect is included.

Interestingly, the sign of the cross-correlation term is preserved between $\mathrm{CbCaTm}$ and $\mathrm{CbCaTb}$ for most amide ${ }^{15} \mathrm{~N}$-spins, despite the different shapes of the associated $\chi$ tensors (prolate for $\mathrm{Tm}^{3+}$ and oblate for $\mathrm{Tb}^{3+}$, Table 1). This is the consequence of the fact that the isotropic part of the $\chi$ tensor is the predominant component contributing to the effective shielding tensor at the site of the nuclear spin.

Overall, the experimental $R_{2}\left({ }^{15} \mathrm{~N}\right)$ PREs tended to be larger than predicted, suggesting the presence of an additional effect influencing the decay of transverse magnetisation in the paramagnetic sample. The deviations are particularly large for nuclear spins with small predicted PREs. The effect could be explained by residual dipolar couplings (RDC) between ${ }^{15} \mathrm{~N}$-spins, which would arise from weak molecular alignment in the magnet caused by the anisotropy of the $\chi$ tensor and, therefore, make the transverse ${ }^{15} \mathrm{~N}$ magnetisation decay faster than expected in the paramagnetic samples. Exchange contributions arising from movements of the bound paramagnetic metal ion also cannot be excluded. ${ }^{23}$

In general, deviations between experiment and predictions are also expected because the calculated cross-correlation effects strongly depend on the exact orientations of the amide bonds, and are therefore sensitive to any uncertainties arising from structural differences between the crystal structure, which is of the wild-type protein, and the solution structure of the double mutant. In addition, we had assumed the same CSA tensor eigenvalues for each amide nitrogen, although it is known that they vary somewhat between different amino acid residues. ${ }^{24}$ 
${ }^{15} \mathrm{~N}$ and ${ }^{13} \mathrm{C}$ PREs have previously been shown to give poor correlations between experimental and calculated values. Specifically, longitudinal ${ }^{15} \mathrm{~N}$ and ${ }^{13} \mathrm{C}$ PREs in $\mathrm{Cu}(\mathrm{II})$ plastocyanin were reported to be uniformly larger than expected at greater distances from the paramagnetic center. ${ }^{25}$ In this case, however, the discrepancies cannot be attributed to DSA-CSA cross-correlation effects, as the electron relaxation rate of $\mathrm{Cu}^{2+}$ was longer than the rotational correlation time of the protein and a different relaxation mechanism applies.

In calbindin $\mathrm{D}_{9 \mathrm{k}}$ under the conditions used in the present work, the greatest ${ }^{15} \mathrm{~N}$ line narrowing effect is predicted for the distance of about $9 \AA$ between the backbone ${ }^{15} \mathrm{~N}$ spins and the paramagnetic centre (see SI and Table S1). At this distance, the much greater PRE of ${ }^{1} \mathrm{H}$ spins would broaden the ${ }^{1} \mathrm{H}$ NMR signals beyond detection, preventing the measurement of very negative PREs by ${ }^{1} \mathrm{H}$-detected experiments. Given the increasing popularity of ${ }^{15} \mathrm{~N}$ direct detection techniques, ${ }^{26,27}$ it is possible that strong negative PREs, leading to very sharp lines, could end up being used for signal enhancement in ${ }^{15} \mathrm{~N}$ NMR spectra, including in situations where amide nitrogens are bound to ${ }^{2} \mathrm{H}$ rather than ${ }^{1} \mathrm{H}$ nuclei.

In conclusion, while it may be tempting to exploit the diversity in paramagnetic strengths of different lanthanide ions to tune the distance range of observable PREs, the present work establishes beyond doubt that PREs must be interpreted with great care when the Curie spin relaxation mechanism is predominant and the observed nuclear spin is endowed with a significant CSA. Compared with ${ }^{15} \mathrm{~N}$, we expect an even greater effect for backbone carbonyl ${ }^{13} \mathrm{C}$ spins due to their larger gyromagnetic ratio and diamagnetic relaxation dominated by the CSA mechanism. It is hard to imagine any alternative methods that could achieve significant line narrowing of carbonyl ${ }^{13} \mathrm{C}$ resonances at high magnetic fields. 


\section{ASSOCIATED CONTENT}

Supporting Information. The following data are available free of charge: ${ }^{15} \mathrm{~N}$ cross-correlated relaxation calculations; protein sample preparation; NMR measurements; tables of chemical shifts, PCS values, and relaxation rates.

\section{AUTHOR INFORMATION}

The authors declare no competing financial interests.

\section{ACKNOWLEDGMENTS}

We thank Prof. Jozef Kowalewski for helpful discussions. H. W. O. acknowledges an Angus Nicholson Honours scholarship. Financial support by the Australian Research Council is gratefully acknowledged.

\section{REFERENCES}

(1) Battiste, J. L.; Wagner, G. Utilization of Site-Directed Spin Labeling and High-Resolution Heteronuclear Nuclear Magnetic Resonance for Global Fold Determination of Large Proteins with Limited Nuclear Overhauser Effect Data. Biochemistry 2000, 39, 5355-5365.

(2) Clore, G. M.; Iwahara, J. Theory, Practice, and Applications of Paramagnetic Relaxation Enhancement for the Characterization of Transient Low-Population States of Biological Macromolecules and Their Complexes. Chem. Rev. 2009, 109, 4108-4139.

(3) Su, X.-C.; Otting, G. Paramagnetic Labeling of Proteins and Oligonucleotides for NMR. J. Biomol. NMR 2010, 46, 101-112. 
(4) Liu, W. M.; Overhand, M.; Ubbink, M. The Application of Paramagnetic Lanthanoid Ions in NMR Spectroscopy on Proteins. Coord. Chem. Rev. 2014, 273-274.

(5) Solomon, I. Relaxation Processes in a System of Two Spins. Phys. Rev. 1955, 99, 559-565.

(6) Bertini, I.; Luchinat, C.; Parigi, G. Solution NMR of Paramagnetic Molecules: Applications to Metallobiomolecules and Models. Elsevier: Amsterdam; London, 2001.

(7) Guéron, M. Nuclear Relaxation in Macromolecules by Paramagnetic Ions: a Novel Mechanism. J. Magn. Reson. 1975, 19, 58-66.

(8) Pintacuda, G.; Kaikkonen, A.; Otting, G. Modulation of the Distance Dependence of Paramagnetic Relaxation Enhancements by CSAxDSA Cross-Correlation. J. Magn. Reson. 2004, $171,233-243$.

(9) Arnesano, F.; Banci, L.; Piccioli, M. NMR Structures of Paramagnetic Metalloproteins. $Q$. Rev. Biophys. 2005, 38, 167-219.

(10) Bertini, I.; Kowalewski, J.; Luchinat, C.; Parigi, G. Cross Correlation Between the DipoleDipole Interaction and the Curie Spin Relaxation: the Effect of Anisotropic Magnetic Susceptibility. J. Magn. Reson. 2001, 152, 103-108.

(11) Bertini, I.; Piccioli, M.; Tarchi, D.; Luchinat, C. COSY Spectra of Paramagnetic Macromolecules: Observability, Scalar Effects, Cross-Correlation Effects, Relaxation-Allowed Coherence Transfer. Concepts Magn. Reson. 1994, 6, 307-335.

(12) Boisbouvier, J.; Gans, P.; Blackledge, M.; Brutscher, B.; Marion, D. Long-Range Structural Information in NMR Studies of Paramagnetic Molecules from Electron Spin-Nuclear Spin Cross-Correlated Relaxation. J. Am. Chem. Soc. 1999, 121, 7700-7701. 
(13) Madhu, P. K.; Grandori, R.; Hohenthanner, K.; Mandal, P. K.; Müller, N. Geometry Dependent Two-Dimensional Heteronuclear Multiplet Effects in Paramagnetic Proteins. $J$. Biomol. NMR 2001, 20, 31-37.

(14) Pintacuda, G.; Hohenthanner, K.; Otting, G.; Müller, N. Angular Dependence of DipoleDipole-Curie-Spin Cross-Correlation Effects in High-Spin and Low-Spin Paramagnetic Myoglobin J. Biomol. NMR 2003, 27, 115-132.

(15) Ghose, R.; Prestegard, J.H. Electron Spin-Nuclear Spin Cross-Correlation Effects on Multiplet Splittings in Paramagnetic Proteins. J. Magn. Reson. 1997, 128, 138-143.

(16) Yao, L.; Grishaev, A.; Cornilescu, G.; Bax, A. The Impact of Hydrogen Bonding on Amide ${ }^{1} \mathrm{H}$ Chemical Shift Anisotropy Studies by Cross-Correlated Relaxation and Liquid Crystal NMR Spectroscopy. J. Am. Chem. Soc. 2010, 132, 10866-10875.

(17) Bertini, I.; Janik, M. B. L.; Lee, Y.-M.; Luchinat, C.; Rosato, A. Magnetic Susceptibility Tensor Anisotropies for a Lanthanide Ion Series in a Fixed Protein Matrix. J. Am. Chem. Soc. 2001, 123, 4181-4188.

(18) Chazin, W. J.; Kördel, J.; Thulin, E.; Hofmann, T.; Drakenberg, T.; Forsén, S. Identification of an Isoaspartyl Linkage Formed upon Deamidation of Bovine Calbindin $\mathrm{D}_{9 \mathrm{k}}$ and Structural Characterization by 2D ${ }^{1} \mathrm{H}$ NMR. Biochemistry 1989, 28, 8646-8653.

(19) Svensson, L. A.; Thulin, E.; Forsén, S. Proline cis-trans Isomers in Calbindin $\mathrm{D}_{9 \mathrm{k}}$ Observed by X-Ray Crystallography. J. Mol. Biol. 1992, 223, 601-606. 
(20) Schmitz, C.; Stanton-Cook, M. J.; Su, X.-C.; Huber, T. Numbat: an Interactive Software Tool for Fitting $\Delta \chi$-Tensors to Molecular Coordinates Using Pseudocontact Shifts. J. Biomol. NMR 2008, 41, 179-189.

(21) Cornilescu, G.; Bax, A. Measurement of Proton, Nitrogen, and Carbonyl Chemical Shielding Anisotropies in a Protein Dissolved in a Dilute Liquid Crystalline Phase. J. Am. Chem. Soc. 2000, 122, 10143-10154.

(22) Vega, A. J.; Fiat, D. Nuclear Relaxation Processes of Paramagnetic Complexes. The Slow-Motion Case. Mol. Phys. 1976, 31, 347-355.

(23) Suturina, E. A.; Kuprov, I. Pseudocontact Shifts from Mobile Spin Labels. Phys. Chem. Chem. Phys. 2016, 18, 26412-26422.

(24) Yao, L.; Grishaev, A.; Cornilescu, G.; Bax, A. Site-Specific Backbone Amide ${ }^{15} \mathrm{~N}$ Chemical Shift Anisotropy Tensors in a Small Protein from Liquid Crystal and Cross-Correlated Relaxation Measurements. J. Am. Chem. Soc. 2010, 132, 4295-4309.

(25) Ma, L.; Jørgensen, A.-M.; Sørensen, G.O.; Ulstrup, J.; Led, J.J. Elucidation of the Paramagnetic $R_{1}$ Relaxation of Heteronuclei and Protons in $\mathrm{Cu}(\mathrm{II})$ Plastocyanin from Anabaena variabilis. J. Am. Chem. Soc. 2000, 122, 9473-9485.

(26) Takeuchi, K.; Arthanari, H.; Shimada, I.; Wagner, G. Nitrogen Detected TROSY at High Field Yields High Resolution and Sensitivity for Protein NMR. J. Biomol. NMR 2015, 63, 323331. 
(27) Takeuchi, K.; Arthanari, H.; Imai, M.; Wagner, G.; Shimada, I. Nitrogen-Detected TROSY Yields Comparable Sensitivity to Proton-Detected TROSY for Non-Deuterated, Large Proteins under Physiological Salt Conditions. J. Biomol. NMR 2016, 64, 143-151. 


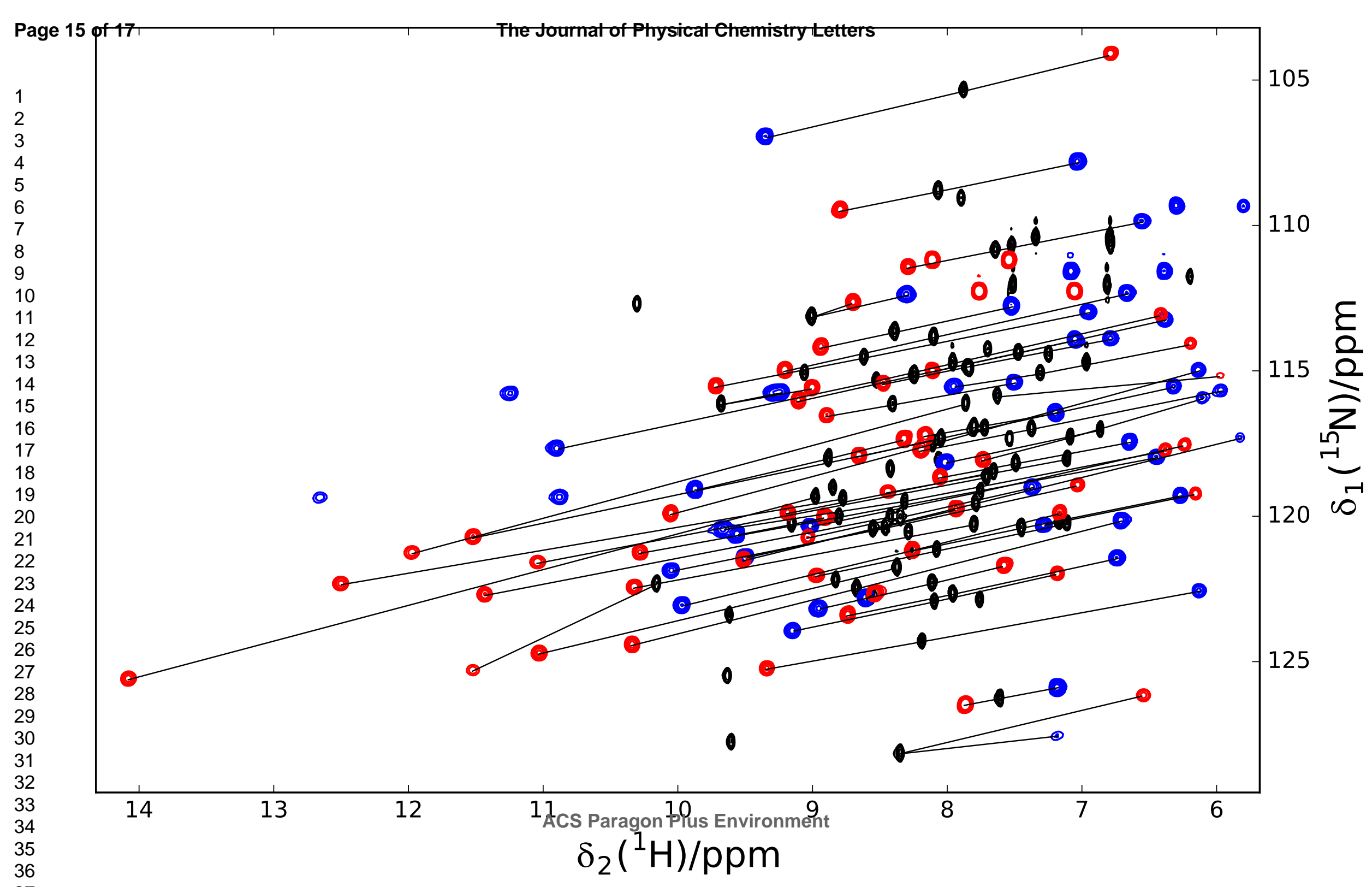




\section{Page 17 of 17 \\ CSA}

The Journal of Physical Chemistry Letters

DSA

1
2
3
4
5
6
7
8
9
1
1
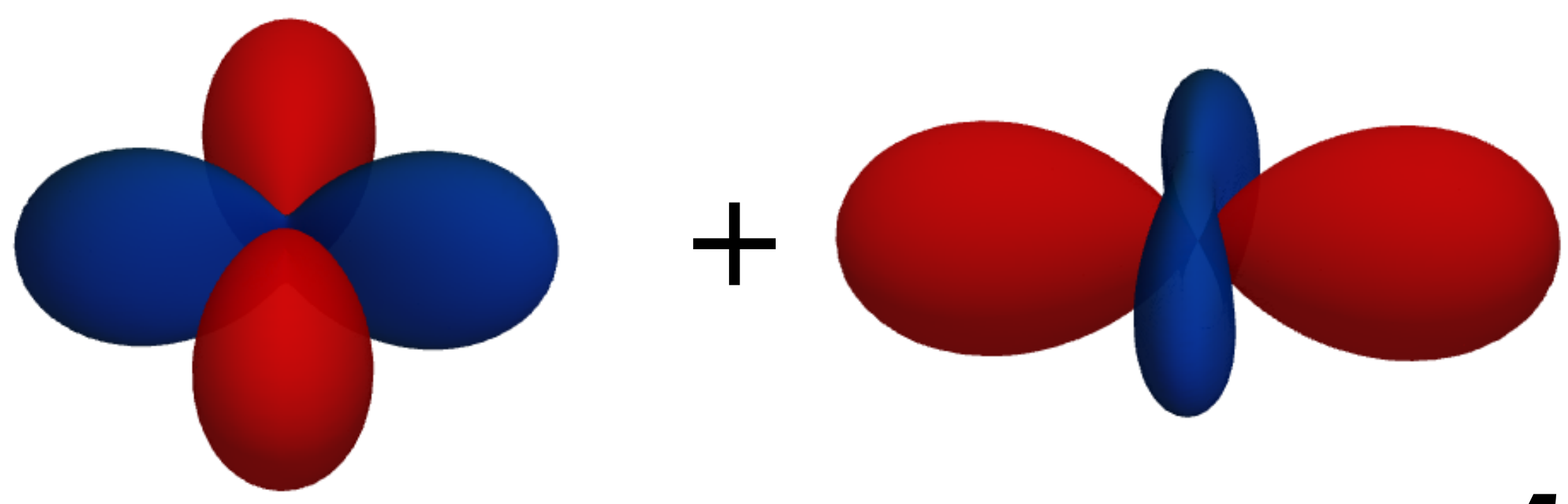

19

24

25

26

27

29

30

31

32

33

34

36

37

38

39

40

41

ACS Paragon Plus Environment

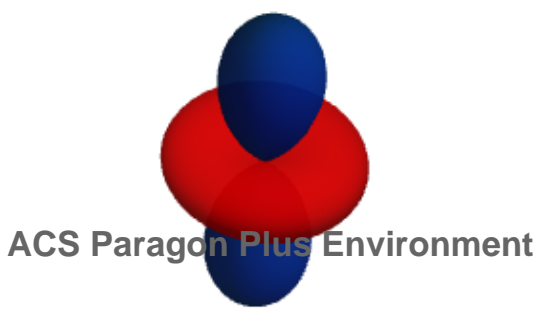

